\title{
Enhancing clinical judgement and decision-making skills of prelicensure nursing students
}

\author{
Megan Kirkpatrick*1, J. David Patterson ${ }^{1}$, Patricia Prince ${ }^{2}$ \\ ${ }^{1}$ University of Calgary, Canada \\ ${ }^{2}$ Mount Royal University, Canada
}

Received: February 1, 2022

Accepted: February 16, 2022

Online Published: February 28, 2022

DOI: $10.5430 /$ jnep.v12n6p45

URL: https://doi.org/10.5430/jnep.v12n6p45

\begin{abstract}
This article describes an authentic learning activity intended to enhance prelicensure nursing students' clinical judgment and decision-making skills. This activity was designed for fourth year nursing students in a pediatric nursing theory course. Part A of the activity requires students to choose the most appropriate staffing assignment for the unit's staff on an inpatient pediatric unit and provide a rationale for that decision. In part B, students review shift reports, prioritize care based on the provided patient information, and identify appropriate referrals to the interdisciplinary team. This activity is adaptable to various educational healthcare disciplines and can be modified for junior and/or senior learners.
\end{abstract}

Key Words: Clinical judgement, Decision-making, Authentic learning, Clinical practice; Pediatric case study

\section{INTRODUCTION}

Registered Nurses (RN) must apply foundational nursing knowledge and clinical judgment skills to provide optimal and safe patient care. Furthermore, they must synthesize assessment findings, understand and work within their scope of practice, and coordinate patient care with the interdisciplinary healthcare team. ${ }^{[1]}$ Pre-licensure nursing students enrolled in a senior pediatric theory course would typically also be enrolled in a pediatric acute care practicum. However, with the arrival of the coronavirus (COVID-19) pandemic, a shift from in-person theory and clinical learning environments to online learning environments occurred. In the spring term of 2020, students were not permitted to attend in-person clinical practice due to the uncertainties involved in the early days of the COVID-19 pandemic. An authentic pediatric learning activity was designed and implemented to support student application of theory-to-practice.
One strategy for enhancing student skills is the use of complex patient care scenarios. The overarching goal of this activity was to promote students' clinical judgment and decision-making skills to identify priorities of care. Specific objectives for the learning activity included:

- Apply foundational nursing knowledge within a pediatric patient and family care environment.

- Analyze patient data, recognize clues, think critically, and tailor nursing care plans accordingly.

- Apply knowledge of pediatric health priorities, trends, and risk mitigation strategies.

- Acknowledge the scope of practice of the interdisciplinary team.

\section{BACKGROUND}

Authentic learning is a student-centred educational technique where learners engage in learning experiences that mirror

*Correspondence: Megan Kirkpatrick; Email: mkirkpatrick@ucalgary.ca; Address: University of Calgary, Canada. 
real-world situations, utilize real-world resources, and promote the use of critical thinking skills. ${ }^{[2]}$ Following this approach, this authentic learning activity was designed to replicate tasks undertaken by RNs in professional practice ${ }^{[3]}$ and to promote student integration of knowledge, skills, and attitudes that they will need to incorporate into their nursing practice.

Nurse educators often find it difficult to measure clinical judgement. According to the National Council of State Boards of Nursing (NCSBN $)^{[4]}$ clinical judgement is "an iterative process that uses nursing knowledge to observe and assess presenting situations, identify a prioritized client concern and generate the best possible evidence-based solutions in order to deliver safe client care" (p.1). Clinical judgment requires critical thinking and decision-making. ${ }^{[4]}$ Muntean ${ }^{[5]}$ reviewed the literature to explore the complexities of novice nurse decision-making and found that decision-making takes more effort and is more deliberate for novice nurses than for experienced nurses. Novice nurses typically take a taskbased approach, following a "to-do" list, versus experienced nurses who consider the "big picture" and can notice subtle changes in their patients. ${ }^{[6]}$ The NCSBN Clinical Judgement Model (NCSBN-CJM) is a foundation for the Next Generation National Council Licensure Exam (NCLEX). ${ }^{[7]}$ The NCLEX tests the competency of entry level nurses, including decision-making skills and clinical judgement. Passing the NCLEX is a licensure requirement for Registered Nurses in Canada and the United States of America. The NCSBN-CJM has divided the process of clinical judgement into four steps: recognizing cues, analyzing cues, prioritizing hypotheses, and generating solutions. ${ }^{[4]}$ The activity discussed in this paper follows this design, providing a foundation to promote and assess students' decision-making and clinical judgement.

\section{LEARNING ACTIVITY}

To provide an authentic learning experience, we developed an individual learning activity that replicates real-world patient and staffing descriptions that students may find on general inpatient and urgent care pediatric units. This case scenario activity consists of two parts (A and B). Part A requires students to choose the most appropriate staffing assignment for the unit's staff on an inpatient pediatric unit and provide a rationale for that decision. RNs are often tasked to create safe and equitable staffing assignments for their unit. Assigning patients to healthcare professionals is a complex evidencebased process that focusses on the desired outcome. ${ }^{[8]}$ In this learning activity students were asked to identify if the care needs matched the scope of practice of the provider they assigned to each patient, and if not, to provide a rationale.

Understanding the roles of other healthcare providers is a crucial component of collaborative practice ${ }^{[9,10]}$ and of providing optimal patient care. To collaborate effectively, healthcare professionals must understand the scope of practice of their colleagues. Students were encouraged to consider the scope of practice of each healthcare provider and possible overlapping scopes of practice. Although there are several models of care, this activity helps develop students' understanding of the complexities involved in creating staffing assignments. Local nursing regulatory bodies determine nurses' scope of practice (RN and Licensed Practical Nurses [LPNs]), therefore, those regulatory guidelines were expected to be followed for this assignment. Students were encouraged to think beyond the basic scope of practice of each healthcare provider and consider the acuity and predictability of each patient, the clinical experience of each provider, advanced competencies of each provider, and possible opportunities for the assigned healthcare providers to collaborate. Students were encouraged to consider if the workload of the staffing assignments was reasonable for each healthcare provider.

In this learning activity, students were asked to choose the most appropriate staffing assignment from three options. The staffing mix and patient complement remained constant, however three different staffing assignment scenarios were offered for students to analyze, make evidence-based decisions, and choose the best assignment. Pediatric inpatient unit staffing complements often includes RNs and LPNs, both of which were included as staffing choices in this assignment. The staff mix for Part A included an RN and two LPNs with varying clinical experience and qualifications. The $\mathrm{RN}$ had 24 years of experience on adult inpatient units (medical, surgical, burns, and plastics) and one year of experience on the general pediatric unit. One LPN had four years of experience on the pediatric unit, working to the full LPN scope of practice, including all advanced competencies. ${ }^{[11]}$ The second LPN completed their final practicum on the same pediatric unit and had worked there since graduation six months ago on a casual basis. The new graduate LPN was not able to perform restricted activities. Students were advised that the charge nurse did not take a patient assignment but was available to cover breaks.

Students were given the following patient information: room number, first and last name, age (ranging from one month to 17 years of age), admitting diagnosis, a brief report, and most recent vital signs. The patient complement ranged from newly admitted complex patients to those awaiting discharge, some with co-morbidities or social determinants of health possibly affecting their care needs. For example, one patient was a 16 year old male who was admitted because of a vasoocclusive crisis secondary to sickle cell disease. The shift report was as follows: 
Pain to extremities; +1 edema to lower limbs; Wt=68kg; PIV NS @ maint; Morphine for pain control via PCA; Naloxone at bedside for rescue; Parents opposed to blood transfusion; Start tinzaparin today; Awaiting serum results; Awaiting CXR; Awaiting Hematology consult; Awaiting Pain Team consult.

Earlier in the course, students had completed a virtual simulation and debrief for a patient with sickle cell disease. Each of the patients listed on the unit linked to specific class content and encouraged further research and critical thinking. Students are generally not encouraged to use acronyms in their practice, however, to provide an authentic learning experience, acronyms were included as they are often used in practice by healthcare providers. To connect the acronyms to a useful learning experience, a list of commonly used acronyms was provided. Students reviewed the information, conducted further research to support their understanding of the patient status, and chose the best assignment between three possible staffing assignment scenarios. Students were asked to provide a detailed rationale for their choice.

Part B of the learning activity further integrated the content of the pediatric-focused senior year theory course. Students were required to review a written shift report for a patient assignment based on a fictitious pediatric urgent care unit. They were then asked to prioritize patient care, identify the main concerns for each patient, and provide the supporting rationale. Students were asked to identify the order in which they would assess their patients (who they would assess first, second, third, etc.) and to provide sound rationale for their prioritization. Students were also asked to identify appropriate referrals to the interdisciplinary care team based on the information provided in the shift report. Students were able to utilize course content and additional resources to complete the activity. The shift report introduction was as follows:

Your shift start time at the pediatric urgent care unit is $0700 \mathrm{~h}$. It's $0700 \mathrm{~h}$ now and you have arrived at the unit and are ready to receive report. The night shift RN left you this written report for your five patients, but they had to leave a couple of minutes ago for a family emergency. Unfortunately, the computer system isn't working and there are no back up charts available at this time.

The intent of this scenario was to encourage students to make informed decisions with the information available and to be adaptable to rapidly changing situations that occur in practice. The patient assignment consisted of five patients ranging from two months to 16 years of age. An example of a patient is as follows:

Age: 5 yrs; Reason for Admission: fever, red blotchy rash on face, cough, conjunctivitis, rhinorrhea; Last Vital Signs: BP 100/65; HR 80; Resps 25; Temp 39.1; O2 Sat 96\% RA; History: Down's Syndrome, AVSD at birth, moderate hearing loss, troubles sleeping. Comments: Unvaccinated, limited supports.

The patient described above was developed to encourage students to think critically about the provided objective data and to research the possible causes of the stated signs and symptoms. The intent of including "limited supports" was to prompt students to consider the patient's support needs and to possibly recommend a referral to the interdisciplinary team.

\section{OUTCOMES}

This learning activity allowed students to apply their knowledge of pediatric care concepts to make clinical judgements and decisions about the care needs of patients. Overall students did well on this assignment, and feedback from the students enrolled in the class was positive. Students stated that the learning activity allowed for a greater understanding of the challenges nurses face when deciding on staffing assignments for the unit. Students also recognized the need for each staff member to be aware of the scope of practice of the entire healthcare team and not just their own personal scope of practice. Students were also able to demonstrate the ability to organize and prioritize patient care with sound supporting rationale. Students in the subsequent practice course (the final practicum) shared that they appreciated the learning from this activity more in hindsight than as the assignment was unfolding. A future consideration for this learning activity is to gather formal feedback to assess the impact on student decision-making and clinical judgement.

Recommendations for educators considering implementing a similar learning activity include: introduce the learning activity in-depth to clarify expectations for students, and hold a fulsome discussion of the scopes of practice of various healthcare providers prior to student participation in this learning activity as this may increase student confidence in their decision-making. Offering subsequent similar activities in class would allow students to build on their learning.

\section{Conclusion}

This authentic learning experience connected pediatric theoretical knowledge to the pediatric practice environs. It allowed students to practice decision-making and fostered the 
development of clinical judgement. The activity ultimately supported the development of student competency to provide safe patient care. The activity offers flexibility for educators and could be utilized as a teaching tool or summative assessment. It could easily be adjusted to be an in-person or online learning activity, or an individual or group assignment. Educators may adapt this case scenario activity for novice

\section{REFERENCES}

[1] Canadian Association of Schools of Nursing. National Nursing Education Framework. 2015. Available from: http: //www . casn. ca/wp-content/uploads/2014/12/F ramwork-FINAL-SB-Nov-30-20151.pdf

[2] Chabeli M, Nolte A, Ndawo G. Authentic learning: A concept analysis. Global Journal of Health Science. 2021; 13(4): 12. https: //doi.org/10.5539/gjhs.v13n4p12

[3] Gulikers JTM, Kester L, Kirschner PA, et al. The effect of practical experience on perceptions of assessment authenticity, study approach, and learning outcomes. Learning and Instruction. 2008; 18(2): 172-186. https://doi.org/10.1016/j.learninstruc .2007 .02 .012

[4] National Council of State Boards of Nursing. Next generation NCLEX News. 2019. Available from: https://www.ncsbn.or g/NGN_Winter19.pdf

[5] Muntean WJ. Nursing clinical decision-making: A literature review. NCSBN Executive Summary. 2012; 1-24. Available from: https://www.ncsbn.org/Clinical_Judgment_Lit_Re view_Executive_Summary.pdf to advanced learners and within various patient populations. Furthermore, educators from various healthcare disciplines may find benefit in applying this type of learning activity as a template to fit their own practice discipline and educational needs.

\section{CONFlicts OF INTEREST Disclosure}

The authors declare that there is no conflict of interest.
[6] Benner P. From novice to expert: Excellence and power in clinical nursing practice. Menlo Park, CA: Addison-Wesley. 1984.

[7] Dickison P, Haerling KA, Lasater K. Integrating the national council of state boards of nursing clinical judgment model into nursing educational frameworks. Journal of Nursing Education. 2019; 58(2): 72-78. PMid:30721306 https : //doi .org/10.3928/01484834-20190 122-03

[8] College and Association of Registered Nurses of Alberta (CARNA). Assignment of Client Care. May. 2014.

[9] Canadian Interprofessional Health Collaborative. CIHC Statement on the definition and principles of Interprofessional Education. 2010; 1-2. Available from: http://www.cihc.ca/files/resources/ CIHCStatement_IPE_Final.pdf

[10] World Health Organization. Framework for Action on Interprofessional Education \& Collaborative Practice. Practice. 2010; 1-63. https://doi.org/10.1111/j.1741-1130.2007.00144.x

[11] College of Licensed Practical Nurses of Alberta. Competency Profile for Licensed Practical Nurses. 5th ed. 2020. 